\title{
Parthenolide exerts inhibitory effects on angiogenesis through the downregulation of VEGF/VEGFRs in colorectal cancer
}

\author{
SE-LIM KIM ${ }^{1,2^{*}}$, SOO-TEIK LEE ${ }^{1,2^{*}}$, KIEU THI THU TRANG ${ }^{1,2}$, SEONG HUN KIM ${ }^{1,2}$, \\ IN HEE KIM ${ }^{1,2}$, SEUNG OK LEE ${ }^{1,2}$, DAE GHON KIM ${ }^{1,2}$ and SANG-WOOK KIM ${ }^{1,2}$ \\ ${ }^{1}$ Department of Internal Medicine, ${ }^{2}$ Research Institute of Clinical Medicine of Chonbuk National University and \\ Biomedical Research Institute of Chonbuk National University Hospital, Jeonju, Jeonbuk 561-712, Republic of Korea
}

Received November 1, 2013; Accepted February 19, 2014

DOI: $10.3892 /$ ijmm.2014.1669

\begin{abstract}
Parthenolide (PT) is responsible for the bioactivities of feverfew (Tanacetum parthenium). Apart from its potent anti-inflammatory effects, this compound has been reported to induce apoptosis in various cancer cells. However, little is known about its role in the process of tumor angiogenesis. In the present study, we investigated the effects and potential mechanisms of action of PT on angiogenesis in human colorectal cancer (CRC). The anti-angiogenic effects of PT were evaluated in cultured human umbilical vein endothelial cells (HUVECs) and in the human CRC cell lines, HT-29, SW620 and HCT116. PT markedly inhibited vascular cell migration and capillary-like structure formation even at a dose which had not effects on cell viability. PT also suppressed the expression of angiogenic biomarker proteins [vascular endothelial growth factor (VEGF), VEGF receptor (VEGFR)1 and VEGFR2] in both the HUVECs and CRC cells. Additionally, PT effectively inhibited tumor neovascularization in a HT-29 xenograft model. These results indicate that PT suppresses angiogenesis by reducing the expression of VEGF and its receptors and may be a viable drug candidate in anti-angiogenesis therapies for human CRC.
\end{abstract}

\section{Introduction}

The process of new blood vessel formation or angiogenesis has become widely recognized as an important target in the treatment of various types of cancer, including metastatic colorectal cancer (mCRC). Folkman's hypothesis of tumori-

Correspondence to: Professor Sang-Wook Kim, Department of Internal Medicine, Chonbuk National University Hospital, 634-18 Geumam-2-dong, Duckjin-gu, Jeonju, Jeonbuk 561-712, Republic of Korea

E-mail: clickm@jbnu.ac.kr

${ }^{*}$ Contributed equally

Key words: parthenolide, colorectal cancer, angiogenesis, human umbilical vein endothelial cells genesis states that tumor growth, invasion and metastasis depend on angiogenesis, which is regulated by pro-angiogenic factors and inhibitors (1-3). Therefore, the blocking of angiogenesis in cancer therapy has become a focus of cancer research. Tumor angiogenesis is closely related with vascular endothelial cells and angiogenesis-related factors. The induction of angiogenesis is mediated by a variety of molecules secreted from cells within tumors. It is well known that vascular endothelial growth factor (VEGF) plays a crucial role in regulating angiogenesis and has become an essential step in anti-angiogenic therapy for the growth and metastasis of cancer $(4,5)$. The agent directly targeting VEGF, bevacizumab, has become part of the standard treatment regimen for $\mathrm{mCRC}$. A number of studies have demonstrated an advantage in favor of the addition of bevacizumab to chemotherapeutic regimens in terms of overall survival, progression-free survival and response rate (6). However, some side-effects attributable to bevacizumab therapy and bevacizumab-resistant tumors have been reported $(7,8)$. Therefore, it is necessary to find a new anti-angiogenic treatment strategy for CRC.

Parthenolide (PT), a natural product, has been used for the treatment of fevers and inflammatory diseases (9). It is responsible for the bioactivities of feverfew (Tanacetum parthenium). Studies have indicated that PT has strong antitumor activities, such as the inhibition of proliferation and the promotion of the apoptosis of tumor cells in various types of cancer (10-14). In a previous study, using xenograft models, we demonstrated that PT may be a potential chemopreventive and therapeutic agent for CRC (15). Moreover, we have demonstrated that combination treatment with PT enhances sensitivity to 5-fluorouracil and promotes apoptosis in CRC (16). However, little has been reported on the effects of PT on vascular endothelial cells from the point of view of tumor angiogenesis. In particular, PT as an anti-angiogenic agent has not been evaluated using an animal model of CRC. Therefore, in this study, we examined whether PT exerts anti-angiogenic effects on human endothelial cells, human CRC cells and in an in vivo mouse model.

\section{Materials and methods}

Chemicals and reagents. PT was obtained from Calbiochem (San Diego, CA, USA). Propidium iodide (PI) was purchased from Sigma-Aldrich (St. Louis, MO, USA). Growth factor- 
reduced Matrigel was purchased from BD Biosciences (San Diego, CA, USA). Mouse recombinant VEGF was purchased from R\&D Systems Inc., (Minneapolis, MN, USA). Anti-VEGF, anti-VEGF receptor (VEGFR)1, anti-VEGFR2 and anti-actin antibodies were obtained from Cell Signaling Technology (Danvers, MA, USA). PT was dissolved in dimethylsulfoxide (DMSO; Sigma-Aldrich) to a concentration of $100 \mu \mathrm{M}$ and stored in the dark at $-20^{\circ} \mathrm{C}$. An aliquot of DMSO was stored under the same conditions and used as a control treatment.

Cell culture and treatment. Human umbilical vein endothelial cells (HUVECs) were purchased from the American Type Culture Collection (ATCC; Rockville, MD, USA) and cultured in endothelial cell growth medium (EGM ${ }^{\circledR}-2 \mathrm{MV}$ Bullet kit; Lonza Inc., Walkersville, MD, USA) containing 2\% fetal bovine serum (FBS). The HUVECs were used at passages 3-4.

The human CRC cell lines, HT-29, SW620 and HCT116, were obtained from ATCC and cultured in RPMI-1640 medium supplemented with $10 \%$ FBS, 100 units penicillin, and 100 units streptomycin. For the treatment of the cells with PT, the cells were subcultured in RPMI-1640 medium without FBS for $12 \mathrm{~h}$. PT dissolved in DMSO was diluted with FBS-free medium to achieve the indicated concentrations. The same concentrations of DMSO were applied to the cells as a control.

3-(4,5-Dimethylthiazol-2-yl)-2,5-diphenyltetrazolium bromide (MTT) colorimetric survival assay. The HUVECs and CRC cells were plated at a density of $1 \times 10^{4}$ cells/well in 96-well plates. The cells were treated with PT for $24 \mathrm{~h}$, the medium was removed, and $200 \mu \mathrm{l}$ of fresh medium plus $20 \mu \mathrm{l}$ of MTT, $2.5 \mathrm{mg}$ dissolved in $50 \mu \mathrm{l}$ of DMSO were added to each well. Following incubation for $4 \mathrm{~h}$ at $37^{\circ} \mathrm{C}$, the culture medium containing MTT was withdrawn and $200 \mu 1$ of DMSO were added, followed by shaking until the crystals were dissolved. Viable cells were determined by measuring the absorbance at $570 \mathrm{~nm}$ using a microplate reader (Molecular Devices, Sunnyvale, CA, USA).

Cell cycle and sub-G1 analysis. Cell cycle and sub-G1 distribution were determined by the staining of DNA with PI (Sigma-Aldrich) [excitation (Ex)/emission (Em), 488/617 nm]. In brief, $1 \times 10^{6}$ cells were incubated with PT for $24 \mathrm{~h}$. The cells were then washed with phosphate-buffered saline (PBS) and fixed in $70 \%$ ethanol overnight. The cells were washed again with PBS and then incubated with PI $(10 \mu \mathrm{g} / \mathrm{ml})$ and simultaneously treated with RNase at $37^{\circ} \mathrm{C}$ for $1 \mathrm{~h}$. The percentages of cells in the different phases of the cell cycle or the sub-G1 DNA content were measured using a BD LSR flow cytometer and analyzed using CellQuest software (Becton Dickinson, New York, NY, USA).

Endothelial cell migration assay: wound healing. Cell migration was evaluated by a wound healing assay, according to the method described in the study by Reischer et al (17). HUVECs $\left(1 \times 10^{6} /\right.$ well) were seeded in 6-cm culture plates and allowed to form a confluent monolayer. The monolayer was then scraped with a P200 pipette tip to generate a wound of approximately $1 \mathrm{~mm}$ in diameter. Subsequently, the medium was changed to fresh endothelial basal medium (EBM-2; supplemented with $20 \mathrm{ng} / \mathrm{ml}$ of VEGF) in the absence or presence of PT. Images of the wounds were captured after $24 \mathrm{~h}$ of incubation at $\mathrm{x} 40$ magnification, and the wound area was determined using an inverted microscope (IX71; Olympus, Center Valley, PA, USA). The ability of the cells to close the wound, that is their motility, was evaluated by determining the healed area. The percentage of the healed area was calculated as follows:

Migration percentage $(\%)=\frac{\begin{array}{c}\text { number of cells in wound area } \\ \text { (group treated with PT) }\end{array}}{\begin{array}{c}\text { number of cells in wound area } \\ \text { (untreated group) }\end{array}} \times 100$

Tube formation assay with HUVECs on Matrigel. Matrigel (200 $\mu 1 /$ well) was added to a 24-well plate and polymerized for $30 \mathrm{~min}$ at $37^{\circ} \mathrm{C}$. The HUVECs $\left(1 \times 10^{4}\right.$ cells/well $)$ were seeded onto each well of the Matrigel-coated 24-well plate and then incubated in EGM-2 containing 2\% FBS. Following overnight incubation, the medium was changed to fresh EBM-2 (supplemented with $20 \mathrm{ng} / \mathrm{ml}$ of VEGF) in the absence or presence of PT. The formation of endothelial cell tubular structure was visualized under an inverted microscope and photographed at $\mathrm{x} 40$ magnification. Furthermore, the tube formation was quantified by calculating the number of polygons and was expressed as a percentage by normalization with PT-untreated control cells.

Cell extraction and western blot analysis. The cells were collected, washed twice with PBS, and then lysed for $30 \mathrm{~min}$ on ice in a lysis buffer $(50 \mathrm{mM}$ Tris- $\mathrm{HCl} \mathrm{pH} 8.0,150 \mathrm{mM}$ EDTA, $1 \%$ Triton X-100, 0.5\% SDS and protease inhibitor cocktail). The protein concentration in the cell lysates was measured using a Protein Quantification kit (Bio-Rad, Hercules, CA, USA). Samples (50 $\mu \mathrm{g}$ protein/well) were loaded onto a SDS-PAGE gel. After transferring and blocking, the membrane was probed with anti-VEGF, anti-VEGFR1, anti-VEGFR2 and anti-actin antibodies. The signal was detected using WEST-one ${ }^{\mathrm{TM}}$ enhanced chemiluminescence solution (Intron Biotechnology Inc., Daejeon, Korea), captured and analyzed by a Luminescent Image Analyzer (LAS-3000; Fujifilm, Tokyo, Japan).

Animal experiments. All animal experiments were approved by the Chonbuk National University Animal Care and Use Committee (Approval no: CBU 2012-0035). The HT-29 $\left(6 \times 10^{6}\right)$ cells were injected into nude mice. The mice were randomly assigned to the control and treatment group and intraperitoneally injected 3 times a week with the vehicle (DMSO) or $4 \mathrm{mg} / \mathrm{kg}$ PT. Treatment with PT or the vehicle commenced 7 days after tumor cell implantation $\left(25 \mathrm{~mm}^{3}\right.$ tumor volume). The experiment was terminated at 28 days, and the tumors were harvested for immunohistochemistry. Immunohistochemistry was carried out in paraffin-embedded $5-\mu \mathrm{m}$-thick tissue sections. The sections were immunostained with an antibody against mouse CD31 (sc-507; Santa Cruz Biotechnology, Inc., Santa Cruz, CA, USA), visualized by appropriate biotin-conjugated secondary antibodies followed by immunoperoxidase detection with the Vectastain ABC Elite kit (Linaris, Dossenheim, Germany) and diaminobenzidine 
(DAB) substrate (Vector Laboratories, Peterborough, UK). Five equal-sized fields were randomly selected. Four fields at x40 magnification were selected and the CD31-positive cells were counted.

Statistical analysis. The data are presented as the means \pm standard error (SE) of at least 3 independent experiments performed in duplicate. Representative blots are shown. All the data were entered into Microsoft Excel 5.0 and SPSS software was used to perform the two-tailed t-tests or the analysis of the variance, where appropriate. P-values $<0.05$ were considered to indicate statistically significant differences.

\section{Results}

Effect of PT on the viability of HUVECs. To determine the anti-angiogenic effects of PT in vitro, we used MTT assay to examine HUVEC viability and survival. Treatment of the HUVECs with PT at a dose of up to $2.5 \mu \mathrm{M}$ did not decrease cell viability. However, at a dose of $>5 \mu \mathrm{M}$ PT, the viability of the HUVECs was significantly reduced in a dose-dependent manner $(\mathrm{P}<0.05)$, showing $2.94 \pm 1.31 \%$ viability at $20 \mu \mathrm{M}$ (Fig. 1A). We also evaluated the effects of PT on cell cycle modifications. After $24 \mathrm{~h}$ of incubation of the HUVECs with or without PT, the cells were analyzed by PI staining and flow cytometric analysis. Treatment with PT resulted in the presence of a sub-G1 cell population, suggestive of cell death. The sub-G1 cell population was also increased in a dose-dependent manner (Fig 1B).

Effects of PT on endothelial cell migration and capillary structure formation. Cell migration is a key step in angiogenesis (18). We therefore investigated the effects of PT on the motility of the endothelial cells by wound healing assay. The results revealed that $\mathrm{PT}$ significantly reduced the migration ability of the HUVECs at $2.5 \mu \mathrm{M}(\mathrm{P}<0.05$; Fig. 2A).

To further investigate the effects of PT on endothelial cells, we examined the VEGF-stimulated tube formation of HUVECs using Matrigel. It is well known that endothelial cells are able to spontaneously form a capillary-like network on Matrigel in vitro, an important feature in the process of angiogenesis (19). HUVECs spontaneously formed a capillary-like tube structure following $24 \mathrm{~h}$ of incubation on Matrigel (Fig. 2B). However, treatment with PT $(2.5 \mu \mathrm{M})$ markedly inhibited the VEGF-stimulated tube formation of the HUVECs, resulting in less elongated, broken and foreshortened tubes.

Effects of PT on the expression of angiogenic biomarkers in HUVECs. VEGF induces endothelial cell proliferation, migration and survival through its cell surface receptors, VEGFR1/VEGFR2 (20). To elucidate the underlying mechanisms responsible for the anti-angiogenic activity of PT, the effects of PT on the expression of VEGF and its receptors in the HUVECs were investigated. Western blot analyses revealed that the treatment of the HUVECs with PT markedly reduced the protein levels of VEGF, VEGFR1 and VEGFR2 compared to the levels in control cells (Fig. 3).

Effects of PT on cell viability and the expression of angiogenic biomarkers in CRC cells. To assess the anti-angiogenic effects

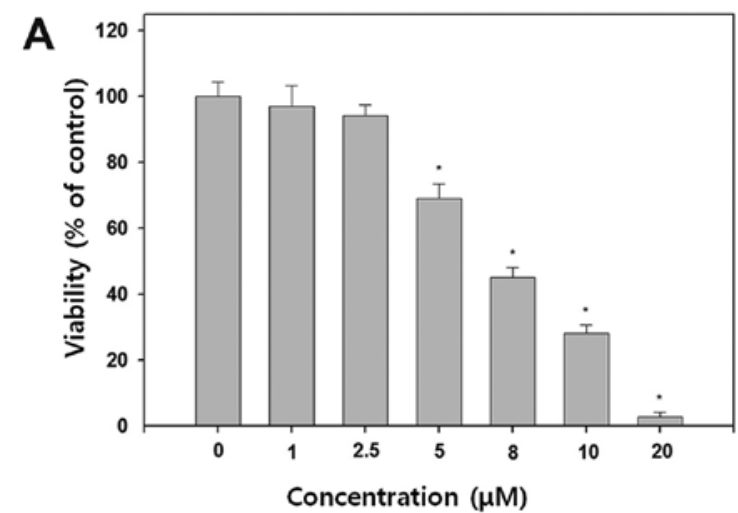

B
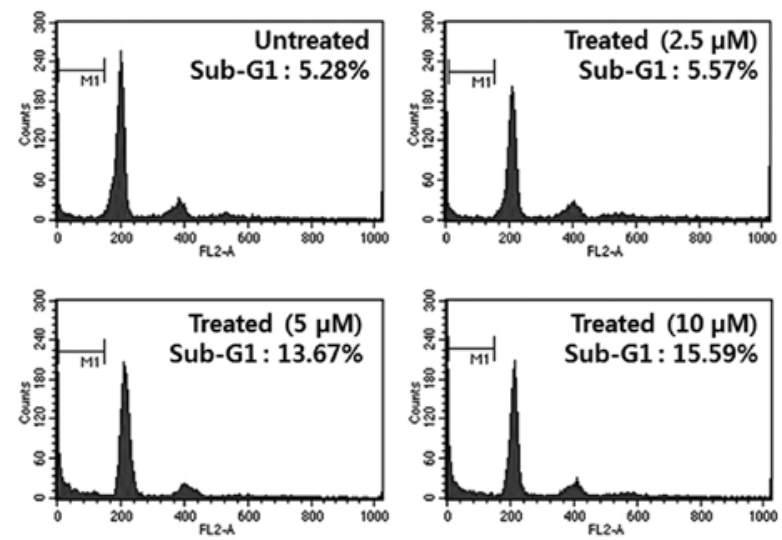

Figure 1. Effect of parthenolide (PT) on the growth of human umbilical vein endothelial cells (HUVECs). (A) Twenty-four hours of exposure to PT resulted in the inhibition of proliferation. Cell viability is expressed as the percentage compared to the vehicle-treated control (100\%). Data are presented as the means \pm standard error (SE) of 3 independent experiments. ${ }^{*} \mathrm{P}<0.05$ vs. control. (B) PT-induced cell death of HUVECs. Flow cytometric detection of apoptosis of HUVECs after $24 \mathrm{~h}$ of exposure to $0,2.5,5$ and $10 \mu \mathrm{M}$ PT. The percentage of the sub-G1 population is shown in each histogram. The experiments were performed at least 3 times and the result of one representative experiment is shown.

of PT in human CRC cells, we investigated its effects on cell viability by MTT assay. The HT-29, SW620 and HCT116 cells were treated with various concentrations $(0,5,10$ and $20 \mu \mathrm{M})$ of PT for $24 \mathrm{~h}$. The viability of these 3 cell lines was decreased in a dose-dependent manner, indicating that the $\mathrm{IC}_{50}$ of $\mathrm{PT}$ is approximately $20 \mu \mathrm{M}$ (Fig. 4A).

We also determined the expression levels of VEGF and its receptors in human CRC cells. In addition, western blot analyses revealed that the levels of VEGF and VEGFR2 were significantly decreased in a dose-dependent manner following treatment with PT (Fig. 4B); the results obtained for the CRC cells are in accord with the results obtained for the HUVECs.

Effect of PT on angiogenesis in vivo. To examine the effects of PT on angiogenesis in vivo, we used a xenograft nude mouse tumor model in which mice were subcutaneously implanted with HT-29 cells. Immunohistochemical analysis of CD31 revealed that the blood vessel network was well developed in the tumors from the control (vehicle-treated) mice, whereas the development of the blood vessel network was substantially 


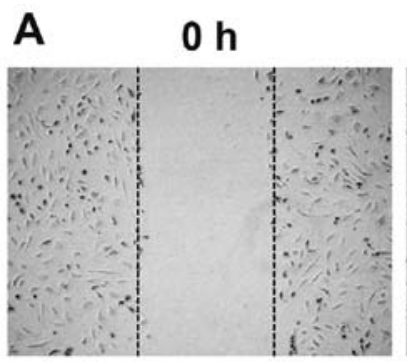

$24 \mathrm{~h}$

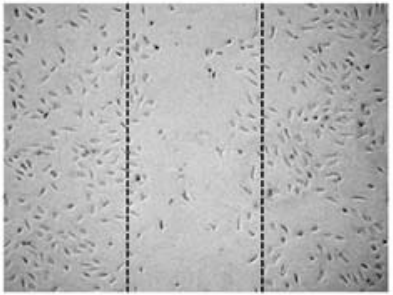

$24 \mathrm{~h}+2.5 \mu \mathrm{M} P T$

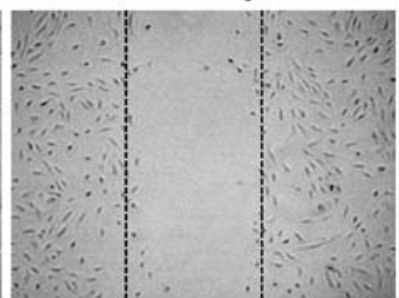

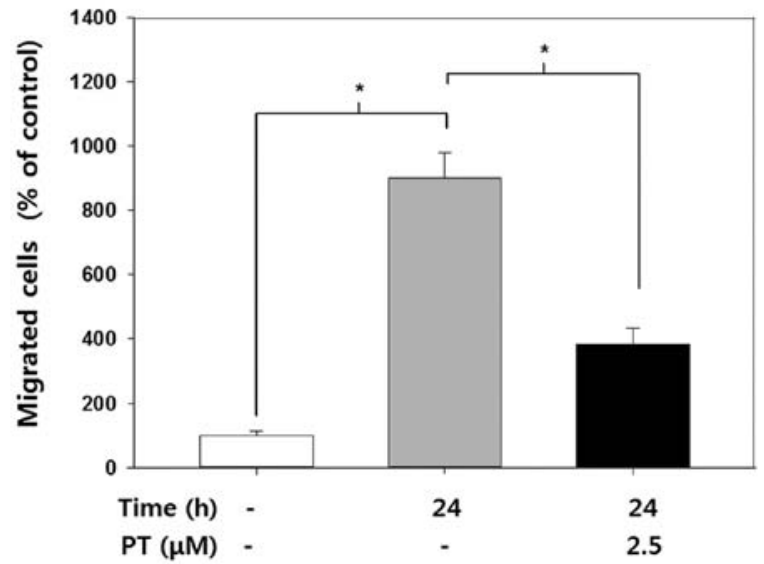
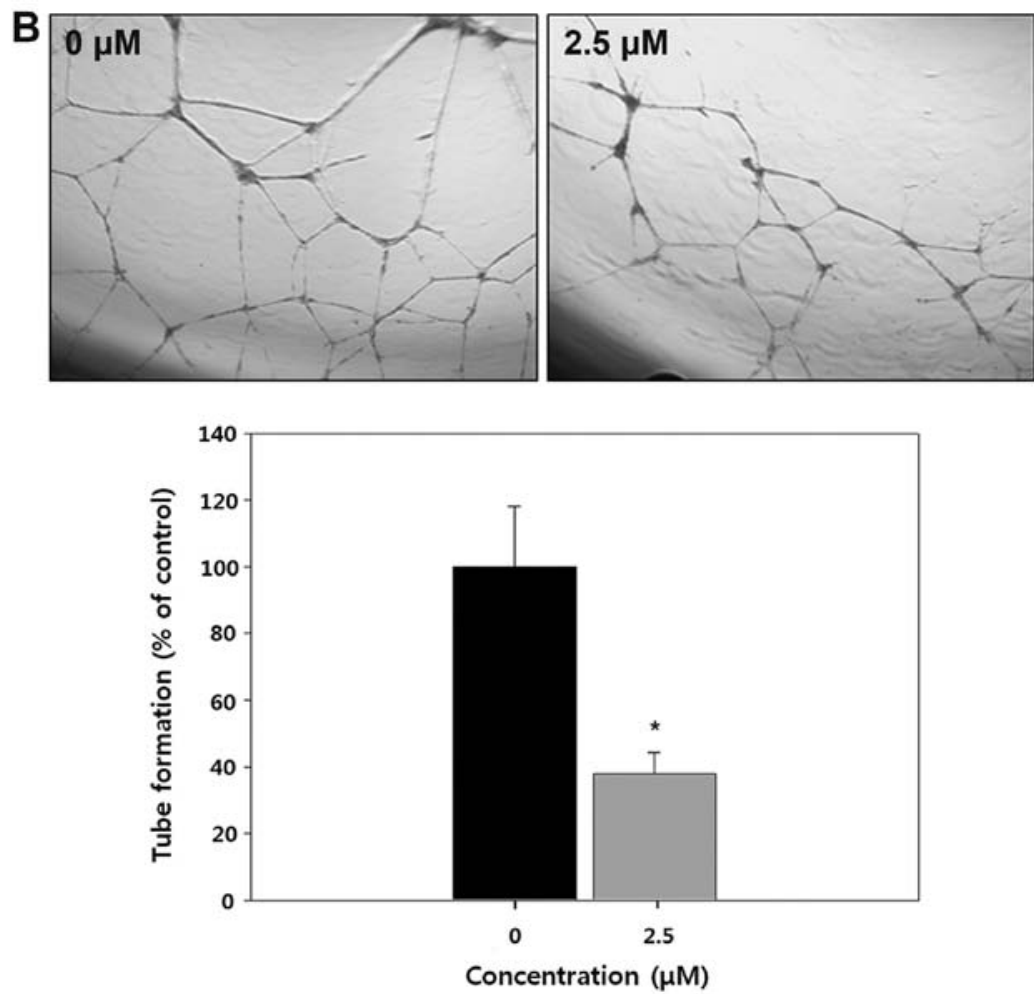

Figure 2. Inhibitory effects of parthenolide (PT) on capillary structure formation and migration of human umbilical vein endothelial cells (HUVECs). (A) PT inhibited HUVEC migration. Cells were grown to confluency in 6-well plates, wounded, and treated with the indicated concentrations of PT for $24 \mathrm{~h}$. The migrated cells were counted. (B) PT inhibited the tube formation of HUVECs. Cells were placed in 24-well plates coated with Matrigel. Following incubation for $4-8 \mathrm{~h}$ in the absence or presence of PT, the tubular structures were photographed. The number of polygons was quantified by manual counting. Data arepresented as the means \pm standard error (SE). ${ }^{*} \mathrm{P}<0.05$.

inhibited by the administration of PT (Fig. 5). Moreover, the tumor tissues from the mice treated with PT displayed a significantly lower number of CD31-positive stained cells, as compared to those from control mice $(\mathrm{P}<0.01)$.

\section{Discussion}

In this study, we investigated the biological functions of PT as an inhibitor of angiogenesis. Our results revealed that PT 


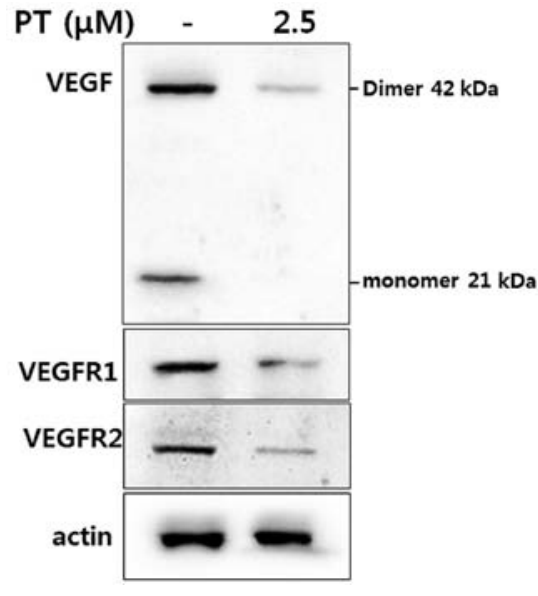

Figure 3. Effects of parthenolide (PT) on the expression of angiogenic biomarkers in human umbilical vein endothelial cells (HUVECs). Cell lysates were prepared following treatment with PT for $24 \mathrm{~h}$ and then analyzed by western blot analysis with vascular endothelial growth factor (VEGF), VEGF receptor (VEGFR)1 and VEGFR2 antibodies. The expression levels of the angiogenic biomarker proteins, VEGF, VEGFR1 and VEGFR2, were significantly decreased following treatment with PT. Actin was used as a loading control.
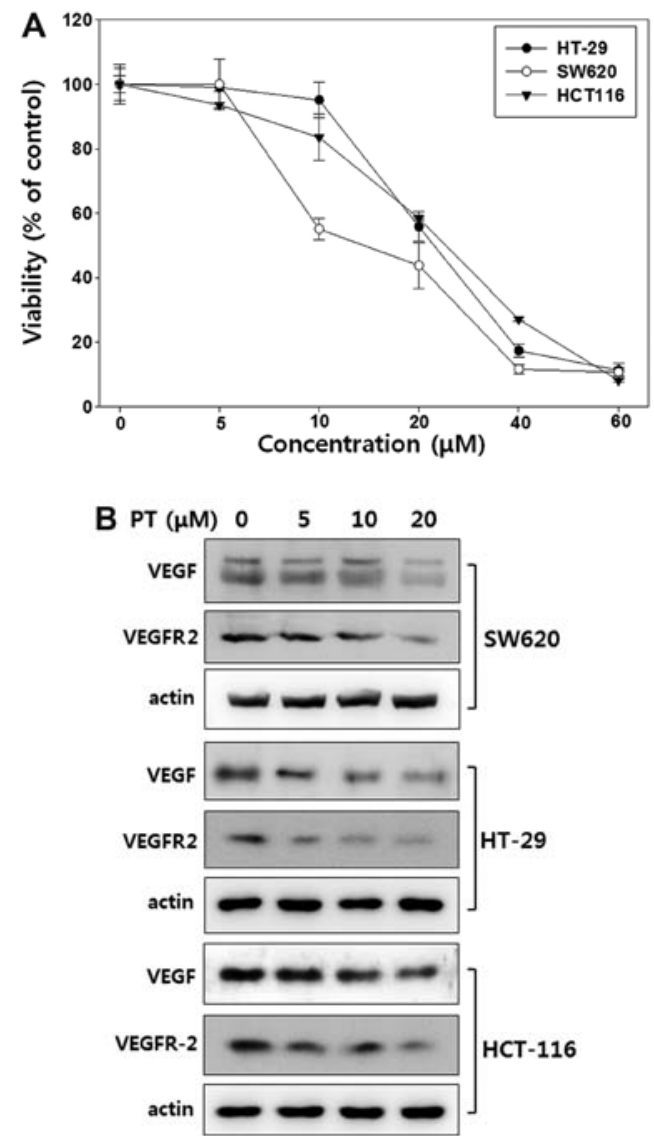

Figure 4. Inhibitory effects of parthenolide (PT) on angiogenesis in human colorectal cancer (CRC) cells. (A) Effect of PT on growth of human CRC cells. Twenty-four hours of exposure to PT resulted in the inhibition of proliferation in a concentration-dependent manner. SW620, HT-29 and HCT116 cell viability is expressed as a percentage compared to the vehicle-treated control and is expressed as the mean \pm standard error (SE). (B) Effects of PT on the expression angiogenic biomarkers. Cell lysates were prepared following treatment with PT for $24 \mathrm{~h}$ and then analyzed by western blot analysis with vascular endothelial growth factor (VEGF) and VEGF receptor (VEGFR)2 antibodies. The expression levels of the angiogenic biomarker proteins, VEGF and VEGFR2, were significantly decreased following treatment with PT in a dose-dependent manner. Actin was used as a loading control.

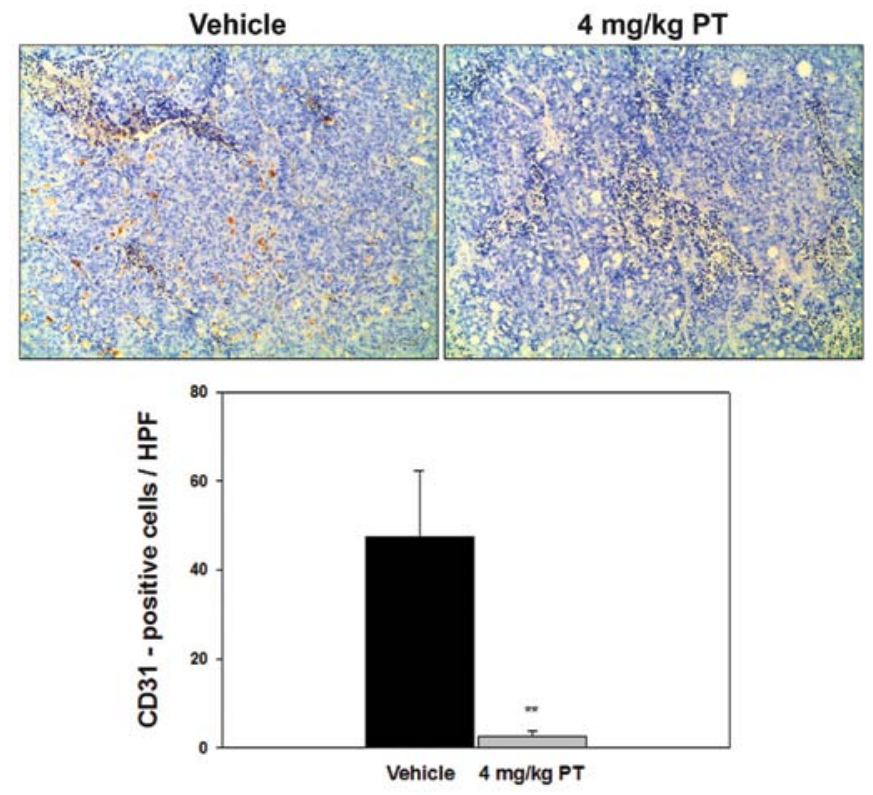

Figure 5. Effects of parthenolide (PT) on angiogenesis in a subcutaneous xenograft tumor model. HT-29 cells were subcutaneously implanted in nude mice. Mice bearing HT-29 tumor xenografts were randomly divided into the vehicle and PT treatment groups. Each treatment group consisted of 6-8 mice treated with the vehicle or $4 \mathrm{mg} / \mathrm{kg}$ PT for 28 days. Tumor sections from animals were fixed and subjected to immunohistochemistry for CD31. Brown spots in the image representing the control (vehicle-treated) mice show positive staining for CD31. The number of CD31-positive cells in tumors was counted at $x 40$ magnification. Columns represent the means \pm standard error $(\mathrm{SE}){ }^{*}{ }^{* *} \mathrm{P}<0.01$ vs. vehicl-treated group.

inhibited various aspects of angiogenesis, including endothelial cell proliferation, migration, capillary structure formation and the expression of angiogenic biomarkers. Moreover, PT markedly reduced the viability of human CRC cells and the protein levels of angiogenic biomarkers in CRC cells. PT also inhibited the tumor volume and angiogenesis in a mouse xenograft tumor model of human CRC $(21,22)$.

$\mathrm{CRC}$ is one of the most common causes of mortality due to malignant tumors worldwide. However, the common therapeutic regimens for CRC, including surgery, chemotherapy and radiation, are insufficient to eliminate the tumor burden and CRC continues to inflict individuals worldwide. Therefore, there is an urgent need to develop novel therapeutic approaches to combat this devastating disease.

A number in vitro studies using PT as a single treatment demonstrated that treatment with 10-100 $\mu \mathrm{M}$ PT for $24 \mathrm{~h}$ resulted in the downregulation of anti-apoptotic genes and in the upregulation of pro-apoptotic genes $(13,15,23-25)$. Of note, in this study, we demonstrated that incubation with PT $(2.5 \mu \mathrm{M})$ for $24 \mathrm{~h}$ markedly inhibited tube formation and migration, as well as the expression of VEGF/VEGFRs in HUVECs. Moreover, the viability of the HUVECs was not affected at this concentration. Our results suggest that the angiogenesis-related genes or pathway respond to low concentrations of PT.

Previous studies, including our own, have suggested the involvement of multiple pathways in PT-induced apoptotic cell death in human cancer cells, and the mechanisms involved include oxidative stress, the inhibition of DNA synthesis, the activation of STAT and nuclear factor $-\kappa \mathrm{B}$ and mitochodrial dysfunction $(10,23,24,26-29)$. However, to our knowledge, 
there is no study available on its ability to inhibit tumor growth in vivo through the inhibition of angiogenesis. In our previous study, we demonstrated an anticancer property of PT using CRC cells and a xenograft tumor model (15). Moreover, we also showed that PT participates in suppressing VEGF expression, using immunohistochemistry for CD31 (15). A study on renal cell carcinoma demonstrated that $\mathrm{PT}$ reduces VEGF production in vitro and in vivo (30). PT has also been shown to inhibit proliferation and induce cell cycle arrest at the G0/ G1 phase in vascular smooth muscle cells (31). Moreover, as previously demonstrated, PT inhibits the proliferation, motility and capillary formation ability of HUVECs and has anti-angiogenic effects on multiple myeloma and breast cancer in vitro $(32,33)$. In this study, we therefore focused on the regulatory effects on angiogenesis and mechanisms of action of PT in CRC. To the best of our knowledge, the present study is the first to demonstrate that PT inhibits angiogenesis through the downregulation of the VEGF/VEGFR pathway.

VEGF, a major regulator of angiogenesis, binds and activates two tyrosine kinase receptors, VEGFR1 (Flt-1) and VEGFR2 (KDR/Flk-1) (34-36). These receptors regulate physiological, as well as pathological angiogenesis. Although VEGFR1 and VEGFR2 are expressed in endothelial cells, it is generally believed that VEGFR2 is the functional receptor that transduces active signals to trigger angiogenic and vascular permeability activity (37,38). VEGFR2 has stronger tyrosine kinase activity than VEGFR1, and transduces the major signals for angiogenesis (39-41). VEGFR2 expression is 3-5-fold higher in the tumor vasculature than in the normal vasculature (42), VEGFR1 is selectively expressed in endothelial cells and macrophagelineage cells (43-46). Thus, VEGFR2 itself and VEGFR2 signaling appear to be critical targets for the suppression of cancer. In our study, treatment with PT inhibited the expression of VEGF and VEGFR1/VEGFR2 in HUVECs. Treatment with PT also reduced the expression of VEGF and VEGFR2 in CRC cells; however, VEGFR1 was not detected in CRC cells. These observations indicate that PT significantly inhibits angiogenesis in CRC through the downregulation of VEGF/VEGFRs mainly in the VEGFR2 signaling pathway. The VEGFR2 signaling pathway involves a number of effectors, including extracellular signal-regulated kinases (ERKs), sarcoma oncogene/ tyrosine kinase (Src), phosphoinositide 3 kinase (PI3K)/Akt, focal adhesion kinase (FAK), protein kinase $\mathrm{C}$ (PKC), phospholipase $\mathrm{C} \gamma(\mathrm{PLC} \gamma)$ and p38 mitogen-activated protein kinase (MAPK) (47-49). However, this requires further investigation, in order to fully elucidate whether PT affects the activation of VEGFR2-mediated downstream signaling.

In conclusion, we found that PT inhibits angiogenesis throught he downregulation of the VEGF/VEGFR signaling pathway which plays multiple roles in regulating neovascularization. Therefore, our results strongly suggest that PT may be an effective antagonist of VEGF/VEGFR-mediated angiogenesis and therefore may be a potential candidate for the development of multifunctional anticancer agents for the treatment of CRC.

\section{Acknowledgements}

This study was supported by the Fund of Biomedical Research Institute, Chonbuk National University Hospital. The authors thank Professor Mie-Jae Im for contributing to and proofreading this manuscript.

\section{References}

1. Folkman J: What is the evidence that tumors are angiogenesis dependent? J Natl Cancer Inst 82: 4-6, 1990.

2. Carmeliet P and Jain RK: Angiogenesis in cancer and other diseases. Nature 407: 249-257, 2000.

3. Folkman J: Tumor angiogenesis: therapeutic implications. N Engl J Med 285: 1182-1186, 1971.

4. Yi ZF, Cho SG, Zhao H, et al: A novel peptide from human apolipoprotein(a) inhibits angiogenesis and tumor growth by targeting c-Src phosphorylation in VEGF-induced human umbilical endothelial cells. Int J Cancer 124: 843-852, 2009.

5. Byrne AM, Bouchier-Hayes DJ and Harmey JH: Angiogenic and cell survival functions of vascular endothelial growth factor (VEGF). J Cell Mol Med 9: 777-794, 2005.

6. Okines A, Puerto OD, Cunningham D, et al: Surgery with curative-intent in patients treated with first-line chemotherapy plus bevacizumab for metastatic colorectal cancer First BEAT and the randomised phase-III NO16966 trial. Br J Cancer 101: 1033-1038, 2009.

7. Hsu JY and Wakelee HA: Monoclonal antibodies targeting vascular endothelial growth factor: current status and future challenges in cancer therapy. BioDrugs 23: 289-304, 2009.

8. Punt CJ and Tol J: More is less - combining targeted therapies in metastatic colorectal cancer. Nat Rev Clin Oncol 6: 731-733, 2009.

9. Knight DW: Feverfew: chemistry and biological activity. Nat Prod Rep 12: 271-276, 1995.

10. Carlisi D, D'Anneo A, Angileri L, et al: Parthenolide sensitizes hepatocellular carcinoma cells to TRAIL by inducing the expression of death receptors through inhibition of STAT3 activation. J Cell Physiol 226: 1632-1641, 2011.

11. Sohma I, Fujiwara Y, Sugita Y, et al: Parthenolide, an NF-kappaB inhibitor, suppresses tumor growth and enhances response to chemotherapy in gastric cancer. Cancer Genomics Proteomics 8: 39-47, 2011.

12. Zanotto-Filho A, Braganhol E, Schröder R, et al: NFאB inhibitors induce cell death in glioblastomas. Biochem Pharmacol 81: 412-424, 2011

13. Zhao LJ, Xu YH and Li Y: Effect of parthenolide on proliferation and apoptosis in gastric cancer cell line SGC7901. J Dig Dis 10: 172-180, 2009.

14. Park JH, Liu L, Kim IH, Kim JH, You KR and Kim DG: Identification of the genes involved in enhanced fenretinideinduced apoptosis by parthenolide in human hepatoma cells. Cancer Res 65: 2804-2814, 2005.

15. Kim SL, Trang KT, Kim SH, et al: Parthenolide suppresses tumor growth in a xenograft model of colorectal cancer cells by inducing mitochondrial dysfunction and apoptosis. Int $\mathrm{J}$ Oncol 41: 1547-1553, 2012.

16. Kim SL, Kim SH, Trang KT, et al: Synergistic antitumor effect of 5-fluorouracil in combination with parthenolide in human colorectal cancer. Cancer Lett 335: 479-486, 2013.

17. Reischer D, Heyfets A, Shimony S, Nordenberg J, Kashman Y and Flescher E: Effects of natural and novel synthetic jasmonates in experimental metastatic melanoma. Br J Pharmacol 150: 738-749, 2007.

18. Shibuya M: Vascular endothelial growth factor (VEGF)Receptor2: its biological functions, major signaling pathway, and specific ligand VEGF-E. Endothelium 13: 63-69, 2006.

19. Tozer GM, Kanthou C and Baguley BC: Disrupting tumour blood vessels. Nat Rev Cancer 5: 423-435, 2005.

20. Meyer RD and Rahimi N: Comparative structure-function analysis of VEGFR-1 and VEGFR-2: What have we learned from chimeric systems? Ann NY Acad Sci 995: 200-207, 2003.

21. Zunino SJ, Ducore JM and Storms DH: Parthenolide induces significant apoptosis and production of reactive oxygen species in high-risk pre-B leukemia cells. Cancer Lett 254: 119-127, 2007.

22. Gopal YN, Chanchorn E and Van Dyke MW: Parthenolide promotes the ubiquitination of MDM2 and activates p53 cellular functions. Mol Cancer Ther 8: 552-562, 2009.

23. Wen J, You KR, Lee SY, Song CH and Kim DG: Oxidative stressmediated apoptosis. The anticancer effect of the sesquiterpene lactone parthenolide. J Biol Chem 277: 38954-38964, 2002. 
24. Zhang S, Ong CN and Shen HM: Critical roles of intracellular thiols and calcium in parthenolide-induced apoptosis in human colorectal cancer cells. Cancer Lett 208: 143-153, 2004.

25. Zhang S, Ong CN and Shen HM: Involvement of proapoptotic Bcl-2 family members in parthenolide-induced mitochondrial dysfunction and apoptosis. Cancer Lett 211: 175-188, 2004.

26. Tacchini L, De Ponti C, Matteucci E, Follis R and Desiderio MA Hepatocyte growth factor-activated NF-kappaB regulates HIF-1 activity and ODC expression, implicated in survival, differently in different carcinoma cell lines. Carcinogenesis 25: 2089-2100, 2004.

27. Dai Y, Guzman ML, Chen S, et al: The NF (nuclear factor)- $\kappa \mathrm{B}$ inhibitor parthenolide interacts with histone deacetylase inhibitors to induce MKK7/JNK1-dependent apoptosis in human acute myeloid leukaemia cells. Br J Haematol 151: 70-83, 2010.

28. Nakshatri H, Rice SE and Bhat-Nakshatri P: Antitumor agent parthenolide reverses resistance of breast cancer cells to tumor necrosis factor-related apoptosis-inducing ligand through sustained activation of c-Jun N-terminal kinase. Oncogene 23: 7330-7344, 2004.

29. Wu C, Chen F, Rushing JW, et al: Antiproliferative activities of parthenolide and golden feverfew extract against three human cancer cell lines. J Med Food 9: 55-61, 2006.

30. Oka D, Nishimura K, Shiba M, et al: Sesquiterpene lactone parthenolide suppresses tumor growth in a xenograft model of renal cell carcinoma by inhibiting the activation of NF-kappaB. Int J Cancer 120: 2576-2581, 2007.

31. Weng SX, Sui MH, Chen S, et al: Parthenolide inhibits proliferation of vascular smooth muscle cells through induction of G0/G1 phase cell cycle arrest. J Zhejiang Univ Sci B 10: 528-535, 2009.

32. Kong F, Chen Z, Li Q, et al: Inhibitory effects of parthenolide on the angiogenesis induced by human multiple myeloma cells and the mechanism. J Huazhong Univ Sci Technolog Med Sci 28: 525-530, 2008.

33. Li CJ, Guo SF and Shi TM: Culture supernatants of breast cancer cell line MDA-MB-231 treated with parthenolide inhibit the proliferation, migration, and lumen formation capacity of human umbilical vein endothelial cells. Chin Med J (Engl) 125: 2195-2199, 2012

34. de Vries C, Escobedo JA, Ueno H, Houck K, Ferrara N and Williams LT: The fms-like tyrosine kinase, a receptor for vascular endothelial growth factor. Science 255: 989-991, 1992.

35. Shibuya M: Role of VEGF-flt receptor system in normal and tumor angiogenesis. Adv Cancer Res 67: 281-316, 1995.

36. Shibuya M: Vascular endothelial growth factor receptor family genes: when did the three genes phylogenetically segregate? Biol Chem 383: 1573-1579, 2002.
37. Ebos JM, Lee CR, Christensen JG, Mutsaers AJ and Kerbel RS: Multiple circulating proangiogenic factors induced by sunitinib malate are tumor-independent and correlate with antitumor efficacy. Proc Natl Acad Sci USA 104: 17069-17074, 2007.

38. Cao Y: Positive and negative modulation of angiogenesis by VEGFR1 ligands. Sci Signal 2: re1, 2009.

39. Shibuya M, Yamaguchi S, Yamane A, et al: Nucleotide sequence and expression of a novel human receptor-type tyrosine kinase gene (flt) closely related to the fms family. Oncogene 5: 519-524, 1990.

40. Sawano A, Takahashi T, Yamaguchi S, Aonuma $\mathrm{M}$ and Shibuya M: Flt-1 but not KDR/Flk-1 tyrosine kinase is a receptor for placenta growth factor, which is related to vascular endothelial growth factor. Cell Growth Differ 7: 213-221, 1996.

41. Kendall RL and Thomas KA: Inhibition of vascular endothelial cell growth factor activity by an endogenously encoded soluble receptor. Proc Natl Acad Sci USA 90: 10705-10709, 1993.

42. Plate KH, Breier G, Weich HA, Mennel HD and Risau W: Vascular endothelial growth factor and glioma angiogenesis: coordinate induction of VEGF receptors, distribution of VEGF protein and possible in vivo regulatory mechanisms. Int J Cancer 59: 520-529, 1994.

43. Kaplan RN, Riba RD, Zacharoulis S, et al: VEGFR1-positive haematopoietic bone marrow progenitors initiate the pre-metastatic niche. Nature 438: 820-827, 2005.

44. Murakami M, Iwai S, Hiratsuka S, et al: Signaling of vascular endothelial growth factor receptor-1 tyrosine kinase promotes rheumatoid arthritis through activation of monocytes/macrophages. Blood 108: 1849-1856, 2006

45. Muramatsu M, Yamamoto S, Osawa T and Shibuya M: Vascular endothelial growth factor receptor-1 signaling promotes mobilization of macrophage lineage cells from bone marrow and stimulates solid tumor growth. Cancer Res 70: 8211-8221, 2010.

46. Kerber M, Reiss Y, Wickersheim A, et al: Flt-1 signaling in macrophages promotes glioma growth in vivo. Cancer Res 68: 7342-7351, 2008

47. Claesson-Welsh L and Welsh M: VEGFA and tumour angiogenesis. J Intern Med 273: 114-127, 2013.

48. Shibuya M: Vascular endothelial growth factor and its receptor system: physiological functions in angiogenesis and pathological roles in various diseases. J Biochem 153: 13-19, 2013.

49. Hicklin DJ and Ellis LM: Role of the vascular endothelial growth factor pathway in tumor growth and angiogenesis. J Clin Oncol 23: 1011-1027, 2005. 